\title{
Efeito agudo do ultrassom focalizado na redução da gordura abdominal sobre a composição corporal, parâmetro metabólico e variáveis cardiovasculares e autonômicas
}

\author{
Acute effect of ultrasound focused on reducing abdominal fat on body composition, metabolic \\ parameter and cardiovascular and autonomic variables \\ Efecto agudo de la ecografía enfocada en reducir la grasa abdominal en la composición corporal, \\ parámetro metabólico y variables cardiovasculares y autonómicas
}

Recebido: 14/04/2021 | Revisado: 21/04/2021 |Aceito: 26/04/2021 | Publicado: 10/05/2021

\author{
Laurinéia da Silva \\ ORCID: https://orcid.org/0000-0001-5134-0249 \\ Toledo Prudente Centro Universitário, Brasil \\ E-mail: laurineiasilva@live.com \\ Laís Manata Vanzella \\ ORCID: https://orcid.org/0000-0002-9494-3143 \\ Universidade Estadual Paulista Júlio Mesquita Filho, Brasil \\ E-mail: laismv@hotmail.com \\ Mayara Ferreira Lima \\ ORCID: https://orcid.org/0000-0002-8017-1808 \\ Universidade do Oestaulista, Brasil \\ E-mail: mayara_limas2@hotmail.com \\ Amanda Meris Nogueira \\ ORCID: https://orcid.org/0000-0001-7122-1474 \\ Universidade do Oeste Paulista, Brasil \\ E-mail: merisnogueira@gmail.com \\ Nathália Soares de Almeida \\ ORCID: https://orcid.org/0000-001-9660-8640 \\ Universidade do Oeste Paulista, Brasil \\ E-mail: nathaliabotuca@gmail.com \\ Rafaela de Araújo Romão Guilhermo \\ ORCID: https://orcid.org/0000-0001-9846-1304 \\ Universidade do Oeste paulista, Brasil \\ Universidade Anhembi Morumbi, Brasil \\ E-mail: raguilhermo@hotmail.com \\ Isabelle Oliveira Leite \\ ORCID: https://orcid.org/ 0000-0002-7992-1480 \\ Universidade do Oeste Paulista, Brasil \\ E-mail: izabelleoliveiraleite@gmail.com \\ Ana Clara Campagnolo Gonçalves Toledo \\ ORCID: https://orcid.org/0000-0001-7660-0449 \\ Universidade do Oeste Paulista, Brasil \\ E-mail: anaclara@unoeste.br
}

\begin{abstract}
Resumo
Objetivo: Analisar a composição corporal, o comportamento de variáveis cardiovasculares e a modulação autonômica de FC após aplicação de ultrassom de alta potência em mulheres com gordura abdominal localizada e excesso de peso. Métodos: Participaram deste estudo 23 mulheres, $(21,3 \pm 3,06$ anos), apresentando pelo menos $1,5 \mathrm{~cm}$ de prega cutânea abdominal, utilizando contraceptivo oral e sedentário. Foi realizada uma visita ao centro estético, onde foram realizadas as avaliações antropométricas iniciais e, antes e após a aplicação da terapia, foram avaliadas a composição corporal (bioimpedância), a pressão arterial (PA) e a avaliação autonômica. Os índices (RMSSD, SDNN) e (HF, LF un e ms2) foram analisados. Para a análise dos efeitos sobre a composição corporal, foram utilizadas variáveis de PA e valores de colesterol e modulação autonômica nos momentos de repouso e recuperação nos momentos pré e pósintervenção, teste t de Student ou teste de Wilcoxon. com pós-teste de Dunn para dados de distribuição não normal, e o tamanho do efeito foi calculado usando Eta-squaredd e p <0,05 para todos os testes. Resultados: O efeito agudo observado após a ultrassonografia focalizada não promoveu diferenças significativas nas comparações pré e pós das variáveis estudadas; entretanto, houve uma tendência no aumento dos índices (SDNN, SD2) e (rMSSD, SD1 e HF ms2 e un) nos momentos de recuperação em relação ao momento de repouso. Conclusão: Uma sessão de terapia ultra-
\end{abstract}


sônica de alta potência não promoveu mudanças significativas na composição corporal, valores de pressão arterial, modulação autonômica dos valores de VFV e colesterol.

Palavras-chave: Gordura abdominal; Composição corporal; Sistema nervoso autônomo; Ultrassom focalizado.

\begin{abstract}
Objective: To analyze the body composition, behavior of cardiovascular variables and autonomic HR modulation after a high power focused ultrasound application in women with localized abdominal fat and overweight. Methods: Twenty-three women participated $(21.3 \pm 3.06$ years $)$, presenting at least $1.5 \mathrm{~cm}$ of abdominal skin fold, using oral contraceptive and sedentary. A visit was made to the aesthetic center, where the initial anthropometric assessments were performed, and before and after the application of the therapy, the body composition (bioimpedance), blood pressure (BP) and autonomic assessment were evaluated. The indices (RMSSD, SDNN) and (HF, LF un and ms2) were analyzed. For the analysis of the effects on body composition, BP variables and cholesterol values and autonomic modulation at rest and recovery moments found in the pre and post-intervention moments, Student's t-test or Wilcoxon test were applied. with Dunn's post-test for non-normal distribution data, and effect size was calculated using Eta-squaredd and p <0.05 for all tests. Results: The acute effect observed after the focused ultrasound therapy did not promote significant differences in the pre and post comparisons of the studied variables; however, there was a tendency in the increase of the indices (SDNN, SD2) and (rMSSD, SD1 and HF ms2 and un) in the moments of recovery compared to the moment of rest. Conclusion: A session of focused high power ultrasound therapy did not promote significant changes in body composition, blood pressure values, autonomic modulation of FV and cholesterol values.
\end{abstract}

Keywords: Abdominal fat; Body composition; Autonomic nervous system; Focused ultrasound.

\title{
Resumen
}

Objetivo: Analizar la composición corporal, el comportamiento de las variables cardiovasculares y la modulación autonómica de la FC tras la aplicación de ecografía de alta potencia en mujeres con grasa abdominal localizada y sobrepeso. Metodos: Participaron de este estudio 23 mujeres (21,3 $\pm 3,06$ años), que presentaban al menos 1,5 cm de pliegue cutáneo abdominal, utilizando anticonceptivos orales y sedentarios. Se realizó una visita al centro de estética, donde se realizaron las valoraciones antropométricas iniciales y, antes y después de la aplicación de la terapia, se evaluó la composición corporal (bioimpedancia), la presión arterial (PA) y valoración autonómica. Se analizaron los índices (RMSSD, SDNN) y (HF, LF un y ms2). Para el análisis de los efectos sobre la composición corporal se utilizaron variables de PA y valores de colesterol y modulación autonómica en los momentos de descanso y recuperación en los momentos antes y después de la intervención, la prueba de la t de Student o la prueba de Wilcoxon. con la prueba posterior de Dunn para datos de distribución no normal, y el tamaño del efecto se calculó utilizando Eta cuadrado y p <0,05 para todas las pruebas. Resultados: El efecto agudo observado después de la ecografía focalizada no promovió diferencias significativas en las comparaciones pre y post de las variables estudiadas; sin embargo, hubo una tendencia al aumento de los índices (SDNN, SD2) y (rMSSD, SD1 y HF ms2 y un) en los momentos de recuperación en relación al momento de descanso. Conclusión: Una sesión de terapia ultrasónica de alta potencia no promovió cambios significativos en la composición corporal, valores de presión arterial, modulación autónoma de VVV y valores de colesterol.

Palabras clave: Grasa abdominal; Composición corporal; Sistema nervioso autónomo; Ecografía focalizada.

\section{Introdução}

A gordura corporal, principalmente na região do abdome, quadris, cintura e flancos, está associada ao sobrepeso e má qualidade de vida. (Nascimento et al., 2018) A diminuição de atividades física fatores genéticos, etnia, idade, gênero, cultura, padrões socioeconômicos, hábitos alimentares, ambiente familiar, escolar e amigos favorecem o aumento do peso. (Zeigler Zachary et al., 2020) esse excesso de peso pode levar a sérias consequências sociais, psicológicas e físicas, sendo associada ao maior risco de morbimortalidade por enfermidades crônicas não transmissíveis, além de aumentar a circunferência abdominal devido ao excesso de adipócitos no tecido subcutâneo (Siqueira \& Maia, 2019). Os adipócitos são células que formam o tecido adiposo (Schetz et al., 2019) e o excesso desse tecido pode promover o acúmulo de gordura subcutânea na região abdominal e ao redor os órgãos, contribuindo para a incidência de distúrbios metabólicos, redução da expectativa de vida e aumento do risco de desenvolvimento de doenças cardíacas, coronarianas, hipertensão, diabetes, osteoartrite e certos tipos de câncer. (Vishvanath \& Gupta, 2019)

Para a redução do tecido adiposo existem tratamentos estéticos invasivos como cirurgias de abdominoplastia, lipoaspiração e mesoterapia. E existe também técnicas manuais como massagens redutoras, tratamentos com eletroterapia 
utilizando aparelhos como o laser, criolipólise, criofrequência, ultrassom, entre outros, e associações com ativos farmacológicos (Junqueira \& Carneiro, 2017). Na eletroterapia, o ultrassom focalizado (HIFU) está entre as técnicas não invasivas na redução da adiposidade localizada. O ultrassom é um aparelho de ação térmica que promove o aumento do fluxo sanguíneo e aumento da elasticidade de estruturas que contém colágeno e também o efeito não térmico que induz a reação orgânica e se manifesta a nível vascular e tecidual todo mecanismo de ultrassom atua com ondas de compressão e descompressão gerando energia positiva e negativa respectivamente no tecido tratado. (Kapoor et al., 2017)

O Ultrassom focalizado de alta intensidade fornece energia que convergem para dentro da área focal, causando um aumento local da cavitação e da temperatura de forma considerável para causar lesão do tecido adiposo subcutâneo com consequente degradação dos adipócitos na área de tratamento, sem danificar os tecidos ao redor. Além dos efeitos térmicos, o HIFU tem um efeito mecânico que perturba as membranas celulares imediatamente, de forma a promover necrose e apoptose celular.

Hiposteniza-se que as alterações metabólicas ao longo da aplicação da terapia de ultrassom focalizado atuem como agente estressor do organismo, que aplicado de maneira aguda possa gerar alterações no comportamento do sistema nervoso central (SNC) e no sistema nervoso autônomo (SNA) a fim de induzir ajustes cardiovasculares com o objetivo de manter a homeostase do organismo. (Catai et al., 2020; Hayano \& Yuda, 2019)

Uma maneira eficiente de avaliar a modulação autonômica cardíaca é a Variabilidade da frequência cardíaca (VFC), que permite a interpretação da interação entre os sistemas simpático e o parassimpático, (Vanderlei et al., 2009; Matsudo et al., 2001) um método não invasivo, de fácil execução e alta reprodutibilidade as análises dos índices da VFC podem ser realizadas por meio de índices lineares, e entre eles destacam-se os índices RMSSD, SDNN no domínio do tempo e os índices de alta e baixa frequência, HF e LF respectivamente no domínio da frequência. (Catai et al., 2020; Hayano \& Yuda, 2019)

Diante disso ressalta-se que a terapia com ultrassom focalizado é indicada na redução de gordura abdominal, porém não está esclarecido na literatura o comportamento do sistema cardiovascular e autonômico desta intervenção quando aplicada de maneira aguda. Assim faz-se importante esclarecer esses aspectos, pois permitindo o uso desta modalidade de intervenção de maneira segura aos seus usuários.

Portanto, hipotetizou para esse estudo que a aplicação do ultrassom focalizado de alta potência na redução da gordura abdominal localizada, devido aos seus efeitos fisiológicos promovidos no tecido adiposo, pode-se gerar influência no sistema cardiovascular, na resposta autonômica e nos valores de colesterol quando aplicado de maneira aguda.

\section{Metodologia}

\subsection{Amostra}

Este foi um estudo de caráter prospectivo e intervencional. Foram entrevistadas 38 mulheres, $(21,3 \pm 3,06$ anos) entre elas 23 foram submetidas a uma sessão de ultrassom focado de alta potência.

As voluntarias apresentaram sobrepeso e acúmulo de gordura abdominal de pelo menos $1,5 \mathrm{~cm}$ de prega cutânea supra ilíaca, em uso de contraceptivo oral e sedentárias, classificadas assim, pelo Questionário Internacional de Atividade Física (IPAQ) como critério de inclusão. (Cardoso \& Baumann, 2016)

Como critério de exclusão, não foram incluídas voluntárias tabagistas, etilistas, e usuárias de medicação que interfira no sistema cardiovascular e respiratório, ser portadoras de doenças cardiovasculares, pulmonares ou metabólicas conhecidas, como também qualquer contraindicação ao uso da terapia de ultrassom focalizado de alta potência. 
Figura 1 - Fluxograma da inclusão das voluntárias da pesquisa.

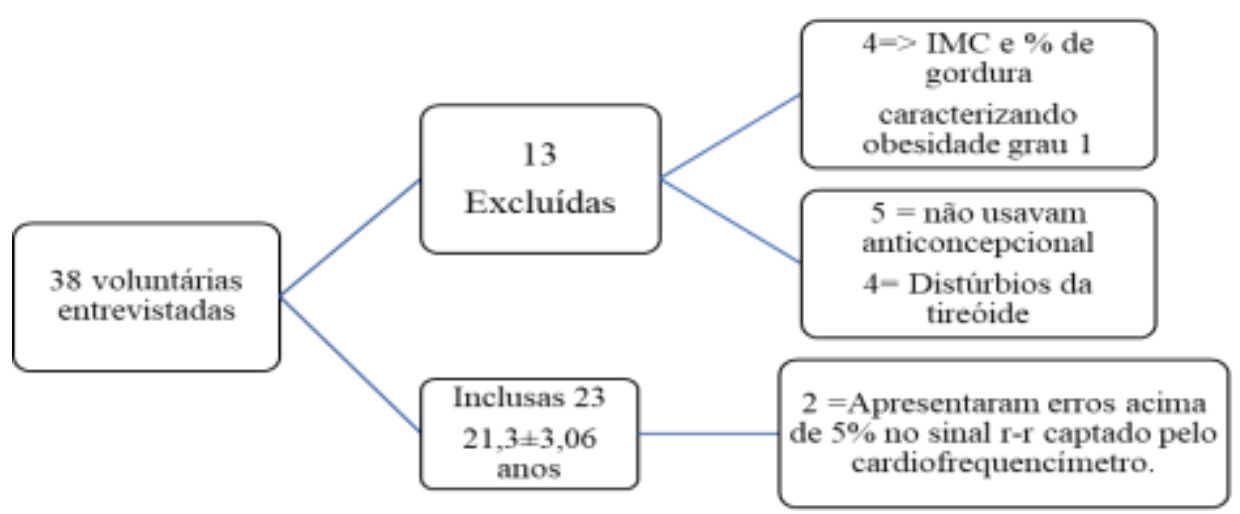

Fonte: Autores.

As voluntárias foram devidamente informadas sobre os procedimentos e objetivos deste estudo e, após concordarem, assinaram um termo de consentimento livre e esclarecido, constituindo a amostra. Os procedimentos utilizados para realização deste trabalho foram avaliados pelo Comitê de Ética em Pesquisa da Universidade do Oeste Paulista - UNOESTE/ Presidente Prudente.

\subsection{Delineamento do estudo}

A coleta dos dados foi realizada na Clínica de Nutrição e Centro de Estética da Universidade do Oeste Paulista (UNOESTE) - Campus 1. Todo o delineamento do estudo foi explicado de maneira individual a cada participante, a fim de esclarecer qualquer dúvida das mesmas antes de iniciar o procedimento experimental.

Todo o procedimento experimental foi realizado em uma sala com controle de temperatura ambiente (temperatura entre $21^{\circ} \mathrm{C}$ e $23^{\circ} \mathrm{C}$ ) e umidade (umidade entre 40 e $60 \%$ ) sempre no período vespertino para evitar as influências do ciclo circadiano sobre as variáveis cardiovasculares.

Todas as voluntárias foram orientadas sobre a importância da abstinência por 24 horas de bebidas que contenham cafeína (café, refrigerante, achocolatados e chás) e álcool. Esses cuidados foram necessários especificamente para avaliações da bioimpedância e do sistema nervoso autônomo antes e após a aplicação da terapia de ultrassom. (Cardoso \& Baumann, 2016)

Antes do início do procedimento experimental as voluntárias foram identificadas com seus dados pessoais.

O procedimento experimental foi realizado com 1 visita das voluntárias a Clínica de Nutrição e Estética, onde foram realizadas as avaliações antropométricas, composição corporal, comportamento autonômico, avaliação dos parâmetros metabólicos (colesterol) por meio da punção da polpa digital e mensurado os valores de pressão arterial, antes e após a aplicação do ultrassom.

Para coleta dos dados da composição corporal, onde foram coletados o índice de massa corporal (IMC), porcentagem de gordura corporal foi utilizado o analisador tetrapolar de Bioimpedância (BIA), esses dados eram captados através de eletrodos descartáveis, posicionados no dorso da mão e do pé do lado direito do corpo das voluntárias.

$\mathrm{Na}$ avaliação antropométrica foi utilizado uma fita métrica corporal, para coletas de dados das circunferências da região abdominal e do quadril.

Em seguida foi realizada a coleta dos valores de prega cutânea, sendo utilizado um adipômetro (Sanny, Brasil) (pressão aproximada de $10 / \mathrm{mm}^{2}$ e divisão de $0,1 \mathrm{~mm}$ ). Esta medida foi realizada no hemicorpo direito da participante na 
posição ortostática, na ausência de contração muscular da região abdominal. A prega abdominal foi pinçada utilizando o dedo polegar e o indicador do avaliador, localizada aproximadamente a $2 \mathrm{~cm}$ à direita da cicatriz umbilical, paralelamente ao eixo longitudinal foi introduzido a extremidade do adipômetro, que após tensão constante de 2 a 3 segundos, foi feita a leitura da dobra em milimetro $(\mathrm{mm})$; essa medida foi repetida por 3 vezes, e foi considerada a média desses valores. (Giorgino et al., 2005)

Para a intervenção deste estudo foi utilizado ultrassom focalizado Sonofocus ${ }^{\circledR}$ (Ibramed, Brasil). O Sonofocus® apresenta frequência de 1,8 MHz modulado por largura de pulso (PWM) em $31 \mathrm{kHz}$ e potência máxima de $30 \mathrm{~W}$. Possui transdutor côncavo que permite a concentração da energia ultrassônica na zona focal, a profundidade controlada (entre 1 a $1,5 \mathrm{~cm}$ ), profundidade ideal para atingir o tecido adiposo sem riscos ao indivíduo. $\mathrm{O}$ tempo de aplicação se deu conforme o tamanho da região tratada de cada participante, e a técnica foi repetida na vertical e horizontal, seguindo as pontuações de demarcação de acordo com o manual do fabricante. O ultrassom focalizado é contraindicado em indivíduos que apresentem camada adiposa subcutânea menor que $1,5 \mathrm{~cm}$, gestantes, dispositivo eletrônico implantado, neoplasias, doenças metabólicas e hepáticas que comprometam o metabolismo das gorduras. (Dyson, 1987)

\subsection{Análise dos parâmetros metabólicos}

Antes e após e a sessão de aplicação do ultrassom focalizado de alta potência foi realizada a medição dos níveis de colesterol, utilizando uma amostra de sangue das participantes, colhida por meio da punção da polpa digital com as tiras de teste correspondente ao aparelho Accutrend Plus (Roche, Brasil), para todas essas análises foram consideradas o período mínimo 4 horas de jejum.

Foram considerados portadores de colesterol total alterado os indivíduos que apresentaram valores $\geq 160 \mathrm{mg} / \mathrm{dl}$, de acordo a IV Diretriz Brasileira sobre Dislipidemias e Prevenção da Aterosclerose. (Andrade, 2003)

\subsection{Avaliação da Variabilidade de Frequência Cardíaca (VFC)}

Para a captação da frequência cardíaca (FC) batimento a batimento foi utilizado o cardiofrequencímetro da marca Polar® S810i (Polar Electro OY, Finlândia), equipamento previamente validado quanto a sua utilização para análise de VFC. (Task, 1996; Ahima \& Flier 2000)

Após a colocação do cardiofrequencímetro, as participantes foram orientadas a permanecerem em repouso na em decúbito dorsal em uma maca, com respiração espontânea durante 20 minutos, pré e pós a aplicação da terapia combinada.

Os dados obtidos por meio da monitorização foram transferidos do cardiofrequequencímetro para o computador por meio do software Polar Precision Performance SW, versão 3.0 e, posteriormente, para cálculo dos índices de VFC foi utilizado o Kubios HRV Standard (Kubios, Biosignal Analysis and Medical Image Group, Department of Physics, University of Kuopio, Finland) (Ahima \& Flier 2000)

Para análise dos dados foram utilizados 256 intervalos RR consecutivos, após ter sido feita filtragem digital complementada por manual, para eliminação de batimentos ectópicos prematuros e artefatos, e somente séries com mais de 95\% de batimentos sinusais foram incluídas no estudo. Foram analisados o tempo de repouso e tempo de recuperação após a aplicação da técnica com ultrassom focalizado, divididos em 4 janelas de tempo de 5 minutos cada. Foram utilizados para análise da VFC índices lineares, obtidos no domínio do tempo (RMSSD e SDNN) e da frequência (LF, HF, LF/HF), em milissegundos e unidades normalizadas. No domínio do tempo foram utilizados os índices RMSSD (à raiz quadrada da média do quadrado das diferenças entre os intervalos RR normais adjacentes em um intervalo de tempo expresso em milissegundos [ms]) e SDNN (desvio padrão de todos os intervalos RR normais gravados em um intervalo de tempo, expresso em $\mathrm{ms}$ ). $\mathrm{O}$ índice SDNN é obtido a partir de registros de longa duração e representam as atividades simpática e parassimpática. Já o índice 
rMSSD representa a atividade parassimpática pois é encontrado a partir da análise de intervalos RR adjacentes. (Ahima \& Flier 2000)

No domínio da frequência, foram utilizados os componentes espectrais de baixa frequência (LF - Frequência entre 0,04 a $0,15 \mathrm{~Hz}$ ), alta frequência ( $\mathrm{HF}$ - Frequência entre 0,15 a $0,4 \mathrm{~Hz}$ ), em unidades normalizadas e em $\mathrm{ms}^{2}$ e a razão entre esses componentes (LF/HF). A análise espectral foi calculada a partir de um tacograma utilizando o algoritmo da transformada rápida de Fourier. (Task, 1996; Ahima \& Flier 2000)

E os índices de atividades globais: SD1, SD2 e a razão SD1/SD2, onde o SD1 representa a dispersão dos pontos perpendiculares à linha de identidade e parece ser um índice de registro instantâneo da variabilidade batimento a batimento; o SD2 representa a dispersão dos pontos ao longo da linha de identidade e representa a VFC em registros de longa duração; a relação de ambos (SD1/SD2) mostra a razão entre as variações curta e longa dos intervalos. (Giorgino et al., 2005)

\subsection{Avaliação da Pressão arterial (PA)}

Para a verificação da pressão arterial foi realizada o método indireto, por meio de um estetoscópio e esfigmomanômetro aneroide fixado no braço esquerdo dos indivíduos. Os valores indicados foram registrados em fichas individuais juntamente com as outras variáveis avaliadas. Para evitar erros na determinação da pressão arterial tais medidas foram realizadas por um único avaliador e os voluntários ficaram sentados em repouso por pelo menos 5 minutos antes da verificação. Foram feitas três aferições com intervalo de 1 minuto entre elas. O valor de PA considerado foi a média das duas últimas aferições, conforme as recomendações de mensuração em consultório contidas na VI Diretrizes Brasileiras de Hipertensão. (Giorgino et al., 2005)

\subsection{Tratamento estatístico}

Para análise dos dados do perfil da população foi utilizado o método estatístico descritivo e os resultados foram apresentados com valores de médias, desvios padrão, mediana, números mínimo e máximo e intervalo de confiança. A normalidade dos dados foi avaliada por meio do teste de teste Kolmogorov-Smirnov.

Nas comparações dos valores de pressão arterial e dos índices da VFC repouso (antes) e recuperação (após) a intervenção foram feitas por meio da técnica de análise de variância para modelo de medidas repetidas. Os dados da mensuração repetida foram checados para violação de esfericidade usando o teste de Mauchly's e a correção de GreenhouseGeisser foi utilizada quando a esfericidade for violada. Para análise dos momentos das variáveis (pré X pós) foi utilizado teste $\mathrm{t}$ de Student para dados pareados (dados paramétricos) ou teste de Wilcoxon (dados não paramétricos). A análise dos valores da pressão arterial sistólica e diastólica antes e após a terapêutica foram comparadas por meio do teste $t$ de Student para dados pareados quando a distribuição for normal ou teste de Wilcoxon para dados com distribuição não normal, com pós-teste de Dunn para dados de distribuição não normal, e o tamanho do efeito foi calculado usando Eta-squaredd e o nível de significância foi estabelecido em $\mathrm{p}<0,05$ para todos testes.

\section{Resultados}

Os dados das características antropométricos das 23 participantes estão apresentados na Tabela 1 em valores de médias, desvio-padrão e intervalo de confiança. 
Tabela 1 - Caracterização do perfil da população do estudo.

\begin{tabular}{|c|c|}
\hline Características pessoais & Inicial \\
\hline Peso (kg) & $\begin{array}{c}75,89 \pm 10,25 \\
(73,5)\end{array}$ \\
\hline Altura (m) & $\begin{array}{c}{[71,46-80,32]} \\
1,62 \pm 0,05 \\
(1,62)\end{array}$ \\
\hline $\operatorname{IMC}\left(\mathrm{Kg} / \mathrm{m}^{2}\right)$ & $\begin{array}{c}{[1,6-1,64]} \\
28,84 \pm 3,29 \\
\quad(27,9)\end{array}$ \\
\hline Perimetria de cintura $(\mathrm{cm})$ & $\begin{array}{c}{[27,42-30,26]} \\
84,19 \pm 5,90 \\
(84)\end{array}$ \\
\hline Perimetria de quadril $(\mathrm{cm})$ & $\begin{array}{c}{[81,64-86,75]} \\
107,48 \pm 7,48 \\
(109)\end{array}$ \\
\hline Perimetria de abdômen (cm) & $\begin{array}{c}{[104,24-10,71]} \\
95,62 \pm 6,72 \\
(96,5)\end{array}$ \\
\hline Prega cutânea supra ilíaca (cm) & $\begin{array}{c}{[92,78-98,46]} \\
5,16 \pm 0,69 \\
(5,1)\end{array}$ \\
\hline Gordura (\%) & $\begin{array}{c}{[4,86-5,46]} \\
27,09 \pm 7,30 \\
\quad(27,3)\end{array}$ \\
\hline $\begin{array}{c}\text { IPAQ - irregularmente ativo A } \\
\text { IPAQ - insuficiente ativo B } \\
\text { IPAQ - sedentário }\end{array}$ & $\begin{array}{c}{[23,93-30,25]} \\
8,69 \% \\
39,13 \% \\
52,17 \%\end{array}$ \\
\hline
\end{tabular}

Legenda: IPAQ - Questionário Internacional de atividade física. Kg: quilogramas, m: metros; cm: centímetro; \%: porcentagem. Fonte: Autores.

Na Tabela 2 estão apresentados a comparação dos valores das variáveis de pressão arterial sistólica e diastólica e colesterol, antes e após a aplicação aguda da intervenção. Não foram observadas alterações nas variáveis de pressão arterial sistólica e diastólica ou para os valores de colesterol total ( $\mathrm{p}>0,05)$. 
Tabela 2 - Valores médios, seguidos dos respectivos desvios padrões, mediana e intervalo de confiança dos valores de pressão arterial sistólica e diastólica e valores de colesterol total, pré e pós a aplicação da terapia.

\begin{tabular}{cccc}
\hline \hline & PAS & PAD & Colesterol \\
\hline \hline Pré & $113,04 \pm 6,35$ & $79,56 \pm 2,08$ & $166,65 \pm 27,14$ \\
& $(110)$ & $(80)$ & $(150)$ \\
& {$[110,3-15,79]$} & {$[78,66-0,47]$} & {$[154,92-78,39]$} \\
Pós & $111,3 \pm 4,7$ & $79,56 \pm 2,08$ & $167,96 \pm 29,58$ \\
& $(110)$ & $(80)$ & $(150)$ \\
& {$[111-115,07]$} & {$[78,66-80,47]$} & {$[155,16-80,75]$} \\
\hline \hline
\end{tabular}

Legenda: PAS: pressão arterial sistólica; PAD: pressão arterial diastólica; mmHg: milímetros de mercúrio. Fonte: Autores.

Os valores dos índices do domínio do tempo, RMSSD e SDNN, antes e após 1 sessão de ultrassom focado de alta potência estão apresentados na Tabela 3. Para ambos os índices em relação ao momento de repouso, há um aumento dos valores ao longo do período de 20 minutos de recuperação, que foi fracionado em 4 janelas de tempo. Contudo não foram observadas diferenças significativas no efeito de momento (RMSSD p> 0,05; SDNN p>0,05).

Tabela 3 - Valores médios, seguidos dos respectivos desvios padrões, mediana e intervalo de confiança dos índices no domínio do tempo (RMSSD e SDNN) antes e depois da aplicação da terapia por ultrassom focado de alta potência.

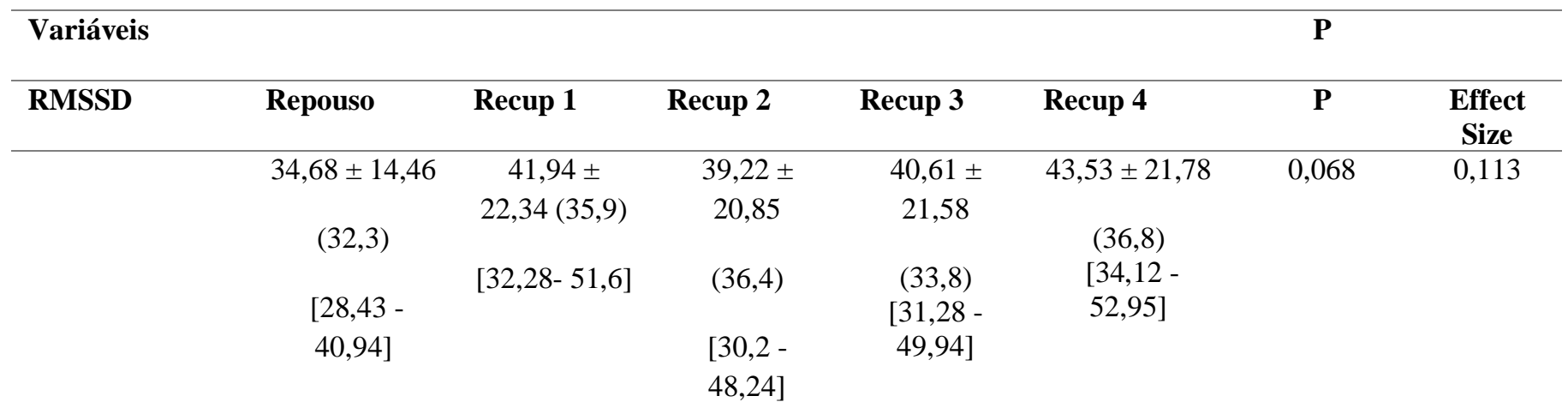

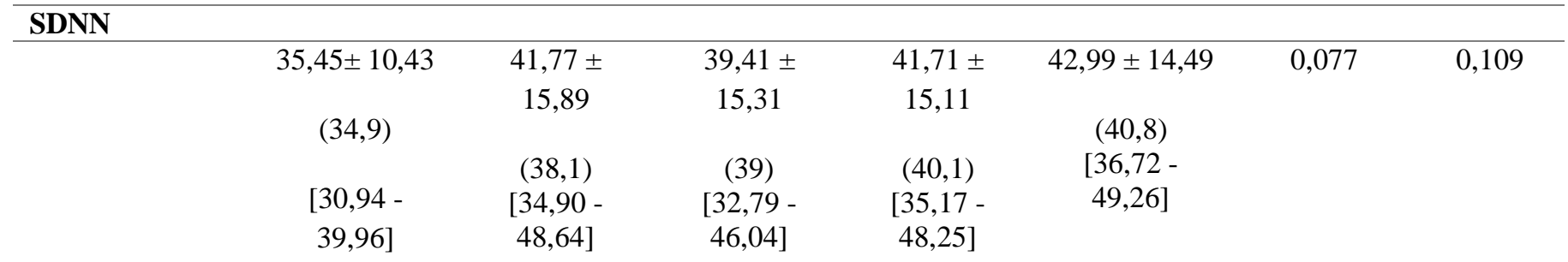

Legenda: RMSSD: raíz quadrada da média da soma dos quadrados de diferença entre intervalos RR adjacente, SDNN: desvio padrão em todos os intervalos RR, Recup: Recuperação. Fonte: Autores.

$\mathrm{Na}$ Tabela 4, estão representados os resultados dos índices espectrais HF e LF em $\mathrm{ms}^{2}$ e un, para esses não foram observadas diferenças significativas no efeito de momento entre os períodos de repouso e recuperação. Mas, é possível observar valores crescentes para os índices HF e LF em ms² nos primeiros 15 minutos de recuperação (fracionados em janelas de 5 minutos), e já nos últimos 5 minutos observa-se diminuição dos valores para todos os índices, sobretudo ainda superior aos valores de repouso (HF e LF ms ${ }^{2}$ p> 0,05); (HF e LF un p>0,05). 
Tabela 4 - Valores médios, seguidos dos respectivos desvios padrões, mediana e intervalo de confiança para os índices no domínio da frequência HF e LF ( $\mathrm{ms}^{2}$ e un) antes e depois da aplicação da terapia por ultrassom focado de alta potência.

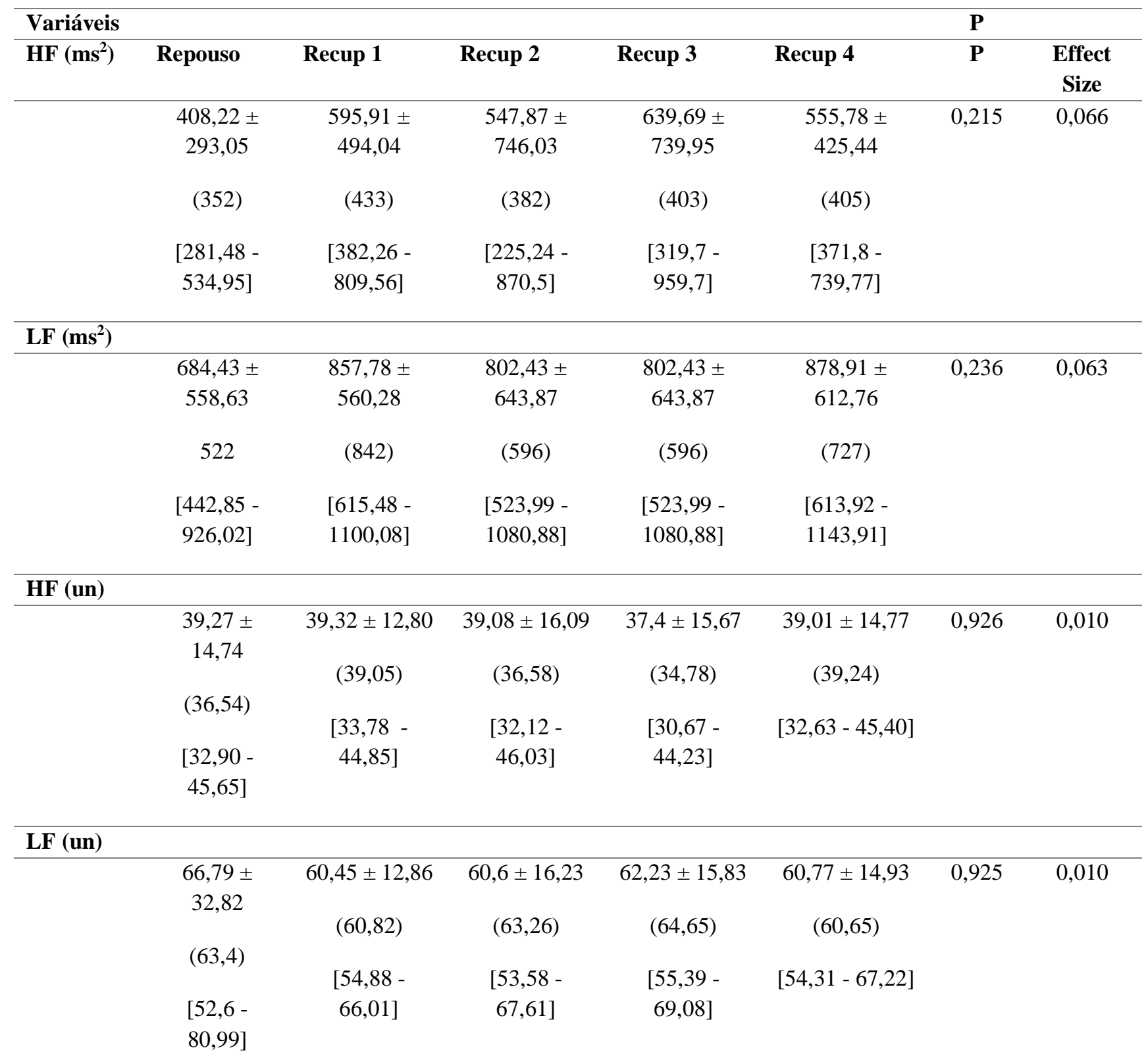

Legenda: HF: poder de alta frequência; LF: poder de baixa frequência, un: unidades normalizadas, $\mathrm{ms}^{2}$ milissegundos ao quadrado, Recup: Recuperação. Fonte: Autores.

Em relação aos índices geométricos SD1 e SD2 para os momentos de repouso e 20 minutos do período de recuperação, estão apresentados os valores médios, seguidos dos respectivos desvios padrão, mediana e intervalo de confiança na Tabela 5. Não havendo efeito imediato, contudo observa-se manutenção do aumento nos valores no período de recuperação (recup 1, recup 2, recup3 e recup 4) em relação ao momento repouso, ( $p>0,05)$. 
Tabela 5 - Valores médios, seguidos dos respectivos desvios padrões, mediana e intervalo de confiança para os índices geométricos SD1 e SD2 pré e pós aplicação da terapia de ultrassom focado de alta potência.

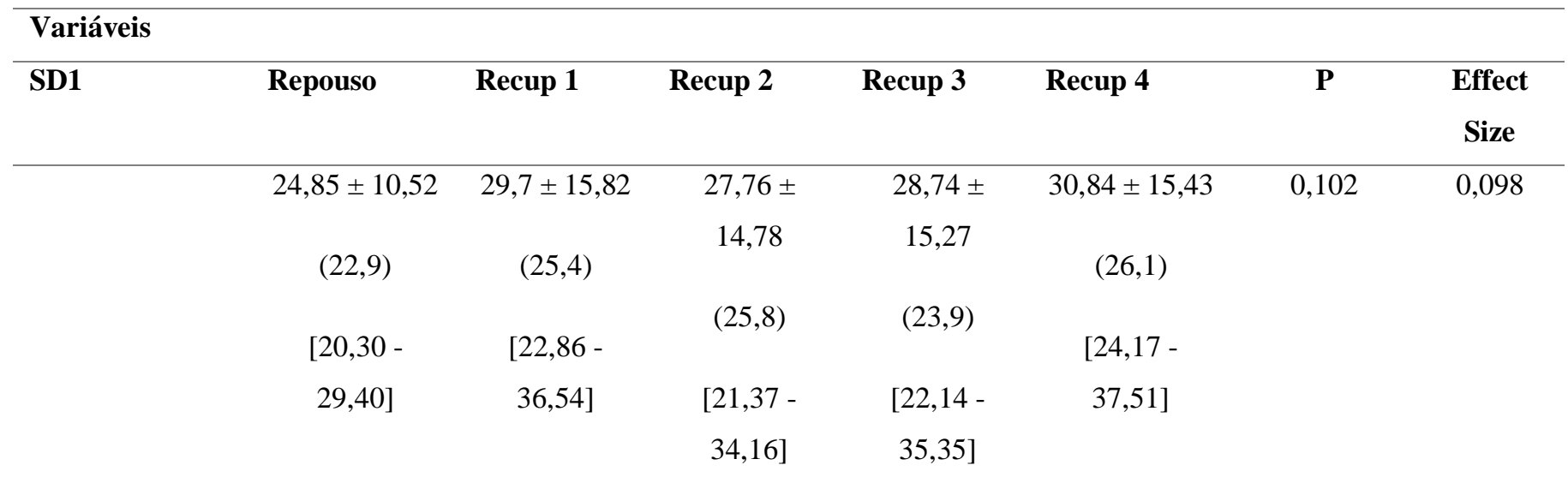

\section{SD2}

\begin{tabular}{|c|c|c|c|c|c|c|}
\hline $42,20 \pm 11,34$ & $50,89 \pm$ & $47,79 \pm$ & $50,95 \pm$ & $51,81 \pm 15,72$ & 0,013 & 0,139 \\
\hline$(40,1)$ & 17,40 & 17,24 & 16,93 & $(48,6)$ & & \\
\hline$[37,30-$ & $(48,5)$ & (45) & $(49,8)$ & {$[45,012-$} & & \\
\hline \multirow[t]{2}{*}{$47,11]$} & {$[43,36-$} & {$[40,33-$} & {$[43,63-$} & $58,61]$ & & \\
\hline & $58,41]$ & $55,24]$ & $58,27]$ & & & \\
\hline
\end{tabular}

Legenda: SD1 desvio padrão batimento a batimento; SD2: Desvio padrão a longo prazo. Recup: Recuperação. Fonte: Autores.

Valores médios, seguidos dos respectivos desvios padrão mediano, intervalo de confiança dos índices no domínio da frequência $(\mathrm{LF} / \mathrm{HF})$ e geométricos (SD1/SD2) encontra-se na Tabela 6. Não foram observadas alterações no momento imediato de repouso, recup1, recup2, recup3 e recup4 ( $\mathrm{p}>0,05)$ para ambos os índices.

Tabela 6 - Valores médios, seguidos dos respectivos desvios padrões, mediana e intervalo de confiança para os índices geométricos SD1/SD2 e do domínio da frequência (LF/HF) pré e pós aplicação da terapia de ultrassom focado de alta potência.

\begin{tabular}{|c|c|c|c|c|c|c|c|}
\hline \multicolumn{8}{|l|}{ Variáveis } \\
\hline \multirow[t]{4}{*}{ SD1/SD2 } & Repouso & Recup 1 & Recup 2 & Recup 3 & Recup 4 & $\mathbf{P}$ & Effect Size \\
\hline & $0,59 \pm 0,19$ & $0,56 \pm 0,16$ & $0,58 \pm 0,18$ & $0,56 \pm 0,19$ & $0,58 \pm 0,19$ & 0,386 & 0,051 \\
\hline & $(0,57)$ & $(0,56)$ & $(0,52)$ & $(0,53)$ & $(0,57)$ & & \\
\hline & {$[0,509-0,67]$} & {$[0,5-0,63]$} & {$[0,5-0,66]$} & {$[0,47-0,64]$} & {$[0,50-0,66]$} & & \\
\hline \multirow[t]{4}{*}{ LF/HF } & Repouso & Recup 1 & Recup 2 & Recup 3 & Recup 4 & $\mathbf{P}$ & Effect Size \\
\hline & $1,96 \pm 1,29$ & $1,82 \pm 0,86$ & $2,06 \pm 1,45$ & $2,32 \pm 1,43$ & $1,98 \pm 1,3$ & 0,197 & 0,054 \\
\hline & $(1,72)$ & $(1,56)$ & $(1,73)$ & $(1,89)$ & $(1,55)$ & & \\
\hline & {$[1,40-2,52]$} & {$[1,45-2,2]$} & {$[1,44-2,69]$} & {$[1,7-2,94]$} & {$[1,42-2,55]$} & & \\
\hline
\end{tabular}

Legenda: SD1/SD2 relação dos índices SD1 e SD2; LF/HF relação dos índices LF e HF; Recup: Recuperação. Fonte: Autores. 


\section{Discussão}

Este estudo avaliou o efeito agudo da aplicação com ultrassom focalizado de alta potência, sobre o comportamento das variáveis cardiovasculares, modulação autonômica e composição corporal de mulheres com sobrepeso. O efeito agudo observado após a intervenção apresentou uma tendência no aumento dos índices da VFC que representam a atividade global (SDNN, SD2) e parassimpática (rMSSD, SD1 e $\mathrm{HF} \mathrm{ms}^{2}$ ), justificada por uma cascata de respostas imediatas fisiológicas e reparadoras do tecido adiposo induzidos pela ação térmica e mecânica do ultrassom, mesmo que essas diferenças não se apresentarem significantes. Contudo esses efeitos sobre a VFC, valores de colesterol e pressão arterial antes e após a aplicação de uma sessão não se mostraram suficientes para induzir alterações.

Os adipócitos são células especializadas no armazenamento de lipídios na forma de triglicerol em seu citoplasma, e essas células possuem enzimas e proteínas reguladoras necessárias para sintetizar e mobilizá-los pela lipólise quando há déficit calórico, essa regulação acontece por meio de nutrientes e sinais aferentes dos tradicionais sistemas neurais e hormonais. (Ghaben \& Scherer, 2019)

Associado a isso, devido ao tecido adiposo participar da liberação de várias moléculas e proteínas com ação de metabolismo local e sistêmica, não tem sido considerado apenas como um reservatório de energia, mas como um órgão com múltiplas funções (Ghaben, 2019) coordenadas pela insulina, cortisol e catecolaminas, participando da regulação de diversos processos como a função endotelial e regulação do balanço energético, por meio de secreção de substâncias como a leptina, TNF- $\alpha$, e adiponectina, entre outras. (Fonseca \& Miriam, 2006; Czech, 2020)

A leptina é um hormônio que atua sinalizando o núcleo arqueado hipotalâmico o qual estimula neuropeptídeos ligados aos mecanismos de inibição da ingestão alimentar e ao aumento do gasto energético através da inervação simpática. Em nível celular, esta proteína ativa a adenil-ciclase e aumenta a oxidação lipídica no músculo esquelético e no fígado, favorecendo a lipólise. (Landecho et al., 2019; Elieh et al., 2020)

Contribuindo no aumento da lipólise e apoptose do adipócito, o TNF- $\alpha$ age diretamente exclusivamente em nível celular e intracelular inibindo acúmulo de gordura, uma vez que interfere nos processos dependentes de insulina (Arner, 1995) na intensão de inibir a lipogênese, mediada por modulação parassimpática, aumentando a lipólise por mecanismos de ação simpática. (Engin, 2017; Elieh, 2020)

A adiponectina é uma adipocina com ação protetora de doenças cardiovasculares e do aumento da sensibilidade insulínica, pois sua ação anti-inflamatória e antiaterogênico, reduz a influência do TNF- $\alpha$, visando retomar o equilíbrio homeostático glicêmico induzindo a lipogênese. (Engin, 2017)

Baseado nas informações supracitadas, entende-se que o SNA influência o metabolismo das células do tecido adiposo por meio de seus intervenientes simpático e parassimpático. A inervação simpática relaciona-se principalmente com as ações catabólicas, tais como a lipólise mediada pelos receptores $\beta$-adrenérgicos e dependente da atividade da enzima lipase hormônio-sensível (LHS) (Saxton et al., 2019) já o sistema nervoso parassimpático está envolvido na execução de efeitos anabólicos sobre os depósitos adiposos, como a captação de glicose e de ácidos graxos estimulada pela insulina. (Goodman, 1970)

Por esses motivos, conjectura-se que aumento desses valores dos índices da variabilidade global, a qual avalia em conjunto as alterações simpáticas e parassimpáticas podem ser justificados devido aos efeitos fisiológicos promovidos pela ação da aplicação do ultrassom focalizado sobre o tecido adiposo. Esses efeitos são decorrentes da ação térmica e mecânica as quais induzem tanto a necrose seletiva, quanto o processo de lipólise dos adipócitos, e secundariamente do aumento do metabolismo local visando a reparação tecidual, todos esses processos são mediados pela ação adrenérgica, podendo atingir um aumento da ação do sistema nervoso autônomo simpático. (D'Ambrosi et al., 2020) 
Na busca do equilíbrio da oferta e demanda de glicose no organismo, a insulina atua tanto na captação periférica de glicose quanto nos tecidos musculares e adiposos, nestes, a insulina estimula a lipogênese no fígado e reduz a lipólise nos adipócitos. (Czech, 2020) por meio do tratamento do ultrassom focado de alta potência atingiu a apoptose e necrose dos adipócitos, fato que implicou no aumento da oferta de triglicerídeos, ácidos graxos e a glicose para a corrente sanguínea, assim acredita-se ter favorecido a predominância parassimpática e o aumento dos índices rMSSD, $\mathrm{HF} \mathrm{ms}^{2}$ e SD1, no período de recuperação da terapêutica e contribuindo também para o incremento dos valores da modulação global e nos valores de colesterol.

Os hábitos alimentares ricos em gorduras e açúcares e sedentarismo, são fatores que reforçam a teoria da lipogênese e da predominância parassimpática. Na prática clínica, para melhores resultados na redução da gordura abdominal localizada, as clientes são orientadas associar dietas alimentares direcionadas por nutricionistas e a prática regular de atividade física, a fim de oferta menos gordura ao metabolismo e também em consumir em forma de energia a gordura retirada do adipócito, com exercícios físicos. (Melero et al., 2020)

O estudo teve limitações que devem ser consideras, como a dificuldade das participantes em permanecerem em jejum por 4 horas e se absterem de alimentos sem cafeína. Perspectiva- se que, um protocolo de tratamento de forma crônica possa trazer mais esclarecimento sobre a ação do ultrassom focalizado sob as variáveis analisadas no presente estudo.

\section{Conclusão}

Diante disso, a lipólise promovida ao tecido adiposo pela ação da aplicabilidade do ultrassom focalizado, promoveu leves alterações a nível de sistema nervoso simpático e a liberação dos ácidos graxos no sangue consequentemente havendo um aumento dos mesmos. Promoveu-se ação no sistema nervoso parassimpático, justificando os possíveis aumentos nos índices do domínio do tempo e da frequência e nos primeiros momentos de recuperação. Este estudo se faz importante para a estética pois é o primeiro a avaliar tais variáveis VFC, colesterol e pressão arterial sob a aplicação do ultrassom focalizado. Entretanto, é necessário mais estudos para esclarecimentos da ação da técnica sob o tecido adiposo e sua relação com a VFC, colesterol e pressão arterial de maneira crônica.

\section{Referências}

Ahima, R. S., \& Flier, J. S. Adipose tissue as an endocrine organ. (2000) Trends Endocrinol Metab 11:327-32

Andrade, J. P. Editores. (2010) VI Diretrizes Brasileiras de Hipertensão. Arquivos Brasileiros de Cardiologia 95(1), 1-51.

Arner, P. (1995) Differences in lipolysis between human subcutaneous and omental adipose tissues. Annals of medicine 27:435-438.

Cardoso, A. A., \& Baumann, L. (2016) Variability of heart rate of individuals and Healthy cyclists at rest and during exercise. Coleção Pesquisa em Educação Física 15(4)

Catai, A. M., Pastre, C. M., Godoy, M. F., Silva, E. D., Takahashi, A., \& Vanderlei, L. (2020). Heart rate variability: are you using it properly? Standardisation checklist of procedures. Brazilian journal of physical therapy, 24(2), 91-102. https://doi.org/10.1016/j.bjpt.2019.02.006

Czech M. P. (2020). Mechanisms of insulin resistance related to white, beige, and brown adipocytes. Molecular metabolism, 34, 27-42. https://doi.org/10.1016/j.molmet.2019.12.014

D'Ambrosi, F., Rossi, G., Soldavini, C. M., Di Maso, M., Carbone, I. F., Cetera, G. E., Colosi, E., \& Ferrazzi, E. (2020). Ultrasound assessment of maternal adipose tissue during 1 st trimester screening for aneuploidies and risk of developing gestational diabetes. Acta obstetricia et gynecologica Scandinavica, 99(5), 644-650. https://doi.org/10.1111/aogs.13800

Dyson, M. (1987) Mechanisms involved in therapeutic ultrasound. Physiotherapy 73 (3), 116- 120.

Elieh Ali Komi, D., Shafaghat, F., \& Christian, M. (2020). Crosstalk Between Mast Cells and Adipocytes in Physiologic and Pathologic Conditions. Clinical reviews in allergy \& immunology, 58(3), 388-400. https://doi.org/10.1007/s12016-020-08785-7

Elieh Ali Komi, D., Shafaghat, F., \& Christian, M. (2020). Crosstalk Between Mast Cells and Adipocytes in Physiologic and Pathologic Conditions. Clinical reviews in allergy \& immunology, 58(3), 388-400. https://doi.org/10.1007/s12016-020-08785-7 
Research, Society and Development, v. 10, n. 5, e38710515064, 2021

(CC BY 4.0) | ISSN 2525-3409 | DOI: http://dx.doi.org/10.33448/rsd-v10i5.15064

Engin A. B. (2017). Adipocyte-Macrophage Cross-Talk in Obesity. Advances in experimental medicine and biology,960, 327-343. https://doi.org/10.1007/978-3-319-48382-5_14

Fonseca, A. \& Miriam, H. (2006) Tecido Adiposo e Regulação Metabólica Arquivos Brasileiros de Endocrinologia e Metabolismo 50(2)

Ghaben, A. L., \& Scherer, P. E. (2019). Adipogenesis and metabolic health. Nature reviews. Molecular cell biology, 20(4), 242-258. https://doi.org/10.1038/s41580-018-0093-Z

Giorgino, F., Laviola, L., \& Eriksson, J. W. (2005). Regional differences of insulin action in adipose tissue: insights from in vivo and in vitro studies. Acta physiologica Scandinavica, 183(1), 13-30. https://doi.org/10.1111/j.1365-201X.2004.01385.x

Goodman, W. (1990) Arthritis and Rheumatism 33(2)

Hayano, J., \& Yuda, E. (2019). Pitfalls of assessment of autonomic function by heart rate variability. Journal of physiological anthropology, 38(1), 3. https://doi.org/10.1186/s40101-019-0193-2

Junqueira, L. C. \& Carneiro, J. (2017) Histologia básica: tecido adiposo 120-124

Kapoor, R., Shome, D., \& Ranjan, A. (2017). Use of a novel combined radiofrequency and ultrasound device for lipolysis, skin tightening and cellulite treatment. Journal of cosmetic and laser therapy: official publication of the European Society for Laser Dermatology, 19(5), 266-274. https://doi.org/10.1080/14764172.2017.1303169

Landecho, M. F., Tuero, C., Valentí, V., Bilbao, I., de la Higuera, M., \& Frühbeck, G. (2019). Relevance of Leptin and Other Adipokines in ObesityAssociated Cardiovascular Risk. Nutrients, 11(11), 2664. https://doi.org/10.3390/nu11112664

Matsudo, S. Araujo, T. Matsudo, V. Andrade, D. Andrade, E. Oliveira, L.C. Braggion, G. Questionario internacional de atividade fisica (IPAQ): Estudo de validade e reprodutibilidade no brasil. (2001) Ativ. Fis. Saúde. 6(2):5-18.

Melero, V., García de la Torre, N., Assaf-Balut, C., Jiménez, I., Del Valle, L., Durán, A., Bordiú, E., Valerio, J. J., Herraiz, M. A., Izquierdo, N., Torrejón, M. J., Runkle, I., Barabash, A., Rubio, M. A., \& Calle-Pascual, A. L. (2020). Effect of a Mediterranean Diet-Based Nutritional Intervention on the Risk of Developing Gestational Diabetes Mellitus and Other Maternal-Fetal Adverse Events in Hispanic Women Residents in Spain. Nutrients, 12(11), 3505. https://doi.org/10.3390/nu12113505

Nascimento, B. R. Brant, L. C. C. \& Oliveira, G. M. M. (2018) Cardiovascular Disease. Epidemiology in Portuguese-Speaking Countries. Arquivos Brasileiros de Cardiologia 110(6), 500-511

Saxton, S. N., Clark, B. J., Withers, S. B., Eringa, E. C., \& Heagerty, A. M. (2019). Mechanistic Links Between Obesity, Diabetes, and Blood Pressure: Role of Perivascular Adipose Tissue. Physiological reviews, 99(4), 1701-1763. https://doi.org/10.1152/physrev.00034.2018

Schetz, M., De Jong, A., Deane, A. M., Druml, W., Hemelaar, P., Pelosi, P., Pickkers, P., Reintam-Blaser, A., Roberts, J., Sakr, Y., \& Jaber, S. (2019). Obesity in the critically ill: a narrative review. Intensive care medicine, 45(6), 757-769. https://doi.org/10.1007/s00134-019-05594-1

Siqueira, K. S. \& Maia, J. M. (2019) Ultrassom terapêutico de alta potência no tratamento da lipodistrofia localizada abdominal - ensaio clínico randomizado duplo cego Universidade Tecnológica Federal do Paraná. Programa de Pós-graduação em engenharia elétrica e informática industrial - CPGEI

Task, F. (1996) Heart rate variability: standards of measurement, physiological interpretation, and clinical use. Europ Heart Journal 17:354-81.

Vanderlei, L. C. M. Pastre, C. M. Hoshi, R. A. Carvalho, T. D. \& Godoy, M. F. (2009) Noções básicas de variabilidade da frequência cardíaca e sua aplicabilidade clínica. Brazilian Journal of Cardiovascular Surgery. 24(2), 205-217.

Vishvanath, L., \& Gupta, R. K. (2019). Contribution of adipogenesis to healthy adipose tissue expansion in obesity. The Journal of clinical investigation, 129(10), 4022-4031.

Zeigler Zachary, F. B., Lopez Brianna, P. G., Welty Jade, D. A., \& Kerekes, M.. (2020). Self-quarantine and weight gain related risk factors during the COVID-19 pandemic. Obesity Research \& Clinical Practice, 3, 210-216. 10.1016/j.orcp.2020.05.004 